\title{
ARTICLE OPEN \\ A fully inkjet-printed disposable glucose sensor on paper
}

\author{
Eloïse Bihar ${ }^{1,2}$, Shofarul Wustoni ${ }^{1}$, Anna Maria Pappa ${ }^{3}$, Khaled N. Salama $\mathbb{D}^{4}$, Derya Baran ${ }^{2}$ and Sahika Inal (iD
}

Inexpensive and easy-to-use diagnostic tools for fast health screening are imperative, especially in the developing world, where portability and affordability are a necessity. Accurate monitoring of metabolite levels can provide useful information regarding key metabolic activities of the body and detect the concomitant irregularities such as in the case of diabetes, a worldwide chronic disease. Today, the majority of daily glucose monitoring tools rely on piercing the skin to draw blood. The pain and discomfort associated with finger pricking have created a global need to develop non-invasive, portable glucose assays. In this work, we develop a disposable analytical device which can measure physiologically relevant glucose concentrations in human saliva based on enzymatic electrochemical detection. We use inkjet-printing technology for the rapid and low-cost deposition of all the components of this glucose sensor, from the electronics to the biorecognition elements, on commercially available paper substrates. The only electronic component of the sensor is the conducting polymer poly(3,4 ethylenedioxythiophene) doped with polystyrene sulfonate (PEDOT:PSS), while the biorecognition element comprises of the enzyme glucose oxidase coupled with an electron mediator. We demonstrate that one month after its fabrication and storage in air-free environment, the sensor maintains its function with only minor performance loss. This fully printed, all-polymer biosensor with its ease of fabrication, accuracy, sensitivity and compatibility with easy-to-obtain biofluids such as saliva aids in the development of next generation low-cost, noninvasive, eco-friendly, and disposable diagnostic tools.

npj Flexible Electronics (2018)2:30; doi:10.1038/s41528-018-0044-y

\section{INTRODUCTION}

Direct access of diagnostic tests at low cost without the need of trained personnel is a major prerequisite in preventative healthcare. Tests that enable self-monitoring and one-shot use are imperative, particularly in low-resource settings where, in most cases, neither sufficient infrastructure nor personnel exist. ${ }^{1}$ Diabetes and cardiovascular diseases are still the major causes of mortality worldwide, with diabetes particularly affecting more than 425 million people every year. ${ }^{2}$ Diabetes is tightly linked with uncontrolled blood sugar levels which lead to a host of complications and damages in vital organs. While in developed countries, screening blood for irregularities is typically performed as part of a routine checkup, measurements of blood glucose are accessible only in the $50 \%$ of the primary care settings in low income countries. ${ }^{3}$ On top of that, the use of a whole-blood sample introduces complications, firstly, regarding the pain and inconvenience that multiple needle pricks cause and secondly the trained personnel required to perform the tests in some cases. Therefore, self-monitoring of critical biomarkers in the body using biofluids alternative to blood, such as saliva and sweat, is in high demand. $^{4-8}$

With the evolution of microelectronics and the possibility of automation, electrochemical sensors have come to fruition demonstrating reliable performance in detecting glucose and real-world applicability (e.g., wearables)..$^{9-11}$ The majority of current electrochemical metabolite sensors rely on the function of enzymes (oxidoreductases). ${ }^{12,13}$ Enzymes are excellent analytical reagents: they reversibly catalyze specific chemical reactions and as such exhibit high sensitivity and selectivity towards certain metabolites. In enzymatic glucose sensors, the biochemical reaction of glucose with the enzyme glucose oxidase generates a change in the electrical current of the electrode nearby that is correlated with glucose concentration. The fabrication of traditional "hard" electronics typically involves tedious and expensive processing methods (such as lithography) due to the nature of the electrode materials used. For the fabrication of sensors wherein application demands are not strictly driven by performance but also by cost-efficiency, for instance those that are designed to be disposable and for single use, additive printing techniques (e.g., inkjet and screen printing) and materials compatible with large area deposition prevail. Inkjet-printing allows for the controlled deposition of a variety of electronic materials in customized geometries and constitutes a low temperature process. ${ }^{14}$ The latter is a feature to be exploited when it comes to the integration of biological molecules, e.g., enzymes, with the electronics, required for biorecognition. ${ }^{15-18}$ Recent advances in the development of organic electronic materials (e.g., conducting polymers) foster the use of printing techniques. The high conductivity and compatibility with screen printing ${ }^{19-21}$ and inkjet-printing, ${ }^{18,22,23}$ render poly(3,4-ethylenedioxythiophene):polystyrene sulfonate (PEDOT:PSS) the benchmark conducting polymer of printed electronics. ${ }^{24}$ Inkjet-printed PEDOT:PSS based devices have been used as biosensors, for instance, for acquiring cutaneous recordings of electrophysiological activity, ${ }^{25-28}$ as well as for enzymatic sensing of gases, ${ }^{22}$ and of glucose. ${ }^{18}$ Due to its soft nature, PEDOT:PSS can also be printed on recyclable, cheap and

\footnotetext{
${ }^{1}$ Division of Biological and Environmental Science and Engineering, King Abdullah University of Science and Technology (KAUST), Thuwal 23955, Saudi Arabia; ${ }^{2}$ Division of Physical Science and Engineering, KAUST Solar Center, KAUST, Thuwal 23955, Saudi Arabia; ${ }^{3}$ Department of Chemical Engineering and Biotechnology, University of Cambridge,

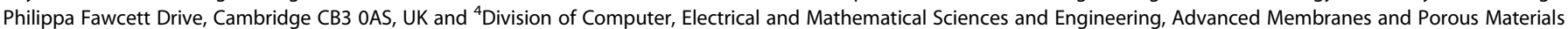
Center, KAUST, Thuwal 23955, Saudi Arabia

Correspondence: Sahika Inal (sahika.inal@kaust.edu.sa)
}

Received: 11 July 2018 Accepted: 15 November 2018

Published online: 03 December 2018 
(a)

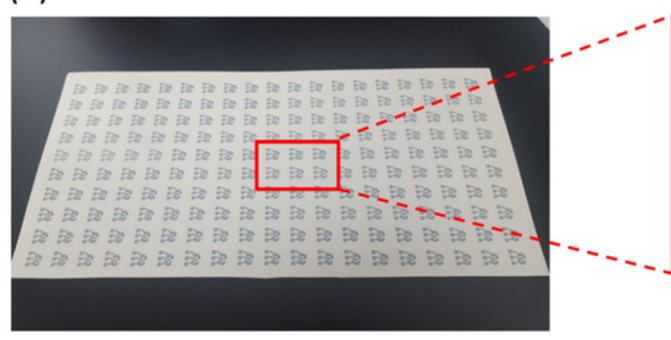

(c) (b)

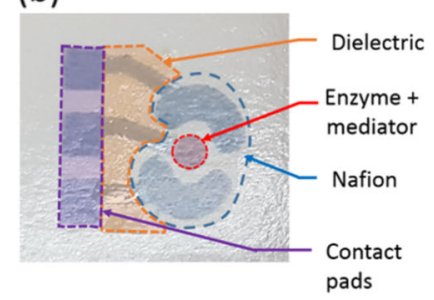

(d)

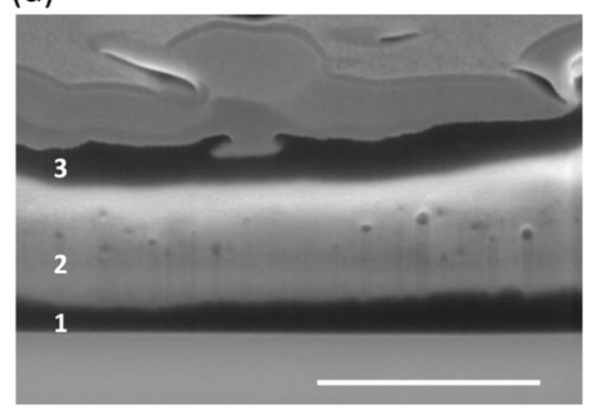

Fig. 1 a Photograph of glucose biosensors inkjet-printed on paper with a loop on the device geometry/configuration. The device is comprised of 2 printed layers of PEDOT:PSS used as reference, working and counter electrode. $\mathbf{b}$ Photograph of the fully printed biosensor. $\mathbf{c}$ 3D exploded schematic of the working electrode with all the separately printed layers, namely the electrode (PEDOT:PSS), the dielectric (SunTronic EMD6415), the biological coating containing the enzyme and the mediator (glucose oxidase and ferrocene), and the encapsulation layer (nafion). d Cross sectional SEM image of the working electrode taken using a focused ion beam (FIB). PEDOT:PSS (1), the biological coating (2), and the encapsulation layer (3) are assembled vertically on paper. The scale bar in a and $\mathbf{d}$ are $1 \mathrm{~cm}$ and $1 \mu \mathrm{m}$, respectively

eco-friendly paper substrates which substitute rather expensive glass and plastics, promising for the realization of disposable in vitro diagnostic tools. ${ }^{29-31}$

In this work, we inkjet-print all the components of an enzymatic glucose sensor on disposable paper substrates. The process consists of four stages of automated deposition of vertical films and is easily transferable to roll-to-roll process. Using only a small quantity of the enzyme and minimizing waste, large quantities of sensors can be fabricated rapidly and at low-cost. Contrary to previously developed electrochemical sensors, the electronics of this sensor consist of $100 \%$ organic materials (i.e., PEDOT:PSS), its biological component is printed and the operation does not require the use of external $\mathrm{Ag} / \mathrm{AgCl}$ electrodes. ${ }^{15,18}$ The device exhibits an operation window down to $0.025 \mathrm{mM}$ and up to $0.9 \mathrm{mM}$ and good sensitivity for glucose concentrations in saliva sufficient for screening abnormal concentrations. When stored at room temperature under vacuum, sensors maintain their function 1 month after fabrication with only minor performance loss $(<25 \%)$. To the best of our knowledge, this is the first report of a metal free, fully inkjet-printed glucose sensor fabricated on a disposable, recyclable paper substrate, which detects glucose in saliva with a shelf life of one month. Inspired by "green electronics", this all-polymer device paves the way for facile, rapid, and low-cost fabrication of biosensors ideal for non-invasive monitoring of metabolites.

\section{RESULTS AND DISCUSSION}

For the fabrication of the device, we used a commercially available PEDOT:PSS ink compatible with inkjet-printing and further optimized the formulation to meet the requirements for the formation of a uniform and conducting layer on paper (see Materials and methods section). The substrate is a commercial glossy paper as shown in Fig. 1a along with the printed PEDOT:PSS features. A three electrode (i.e., reference, working and counter) cell configuration was used to measure the concentration of glucose present in the biological media. Current electrochemical sensors for metabolites typically involve $\mathrm{Ag} / \mathrm{AgCl}$ and Pt electrodes as reference and counter electrodes, respectively. In our device, all the electronic components including the contact pads are composed of the same material, i.e., printed PEDOT:PSS. The electrical conductivity of the conducting polymer ink was found to be $250 \mathrm{~S} / \mathrm{cm}$. In order to insulate/separate the active area from the contact pads area, one layer of a UV-curable dielectric ink was printed on top of the electrode interconnects (Fig. 1b, c). For the incorporation of the biorecognition element, we printed an aqueous solution containing glucose oxidase (GOx) and a ferrocene $(\mathrm{Fc})$ complex on top of the working electrode. $\mathrm{Fc}$ is an electron mediator commonly used in enzymatic sensors as a cosubstrate to replace oxygen. It molecularly wires the enzyme to the sensing electrode, therefore improves the selectivity as well as the operation window of the sensors. However, as Fc adsorbs weakly onto surfaces by itself, and its leakage can raise toxicity concerns, we apply Fc in a solution mixed with the polysaccharide, chitosan. While entrapping Fc within its biocompatible matrix, chitosan improves the processability of the enzyme. The resulting ink was printed on top of the working electrode (Fig. 1b, c). Ultimately, we printed a thin layer of nafion over the sensing area comprising of the working, counter and reference PEDOT:PSS electrodes. As a polyanion, nafion acts as a barrier for the interfering species present in complex biological milieu or formed as a result of unspecific redox reactions during electrode operation. ${ }^{32}$ Figure $1 \mathrm{~d}$ shows a cross sectional SEM image of a typical working electrode. The image suggests incorporation of all the vertical layers of the sensor where PEDOT:PSS, biological coating and nafion layer have a thickness of $160 \mathrm{~nm}, 655 \mathrm{~nm}$, and $190 \mathrm{~nm}$, respectively. As the working electrode is built as a layerby-layer assembly, we examined the morphology and chemical composition of each layer. While the surface of PEDOT:PSS film on paper is relatively featureless, upon the addition of the biological ink and thereafter of nafion, the surface microstructure undergoes large changes (Fig. 2a-c). High resolution X-ray Photoelectron 
(a)

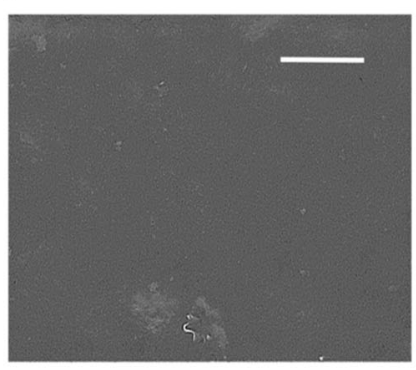

(b)

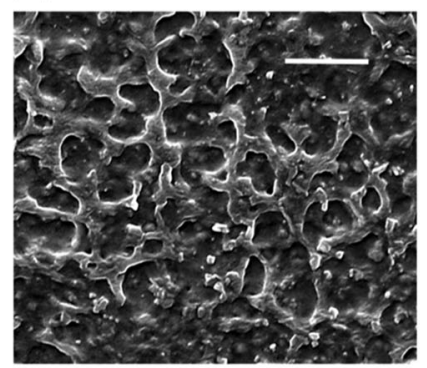

(c)

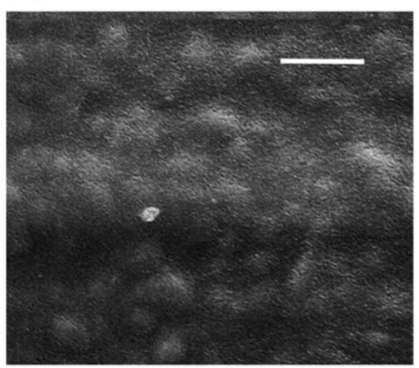

(d)

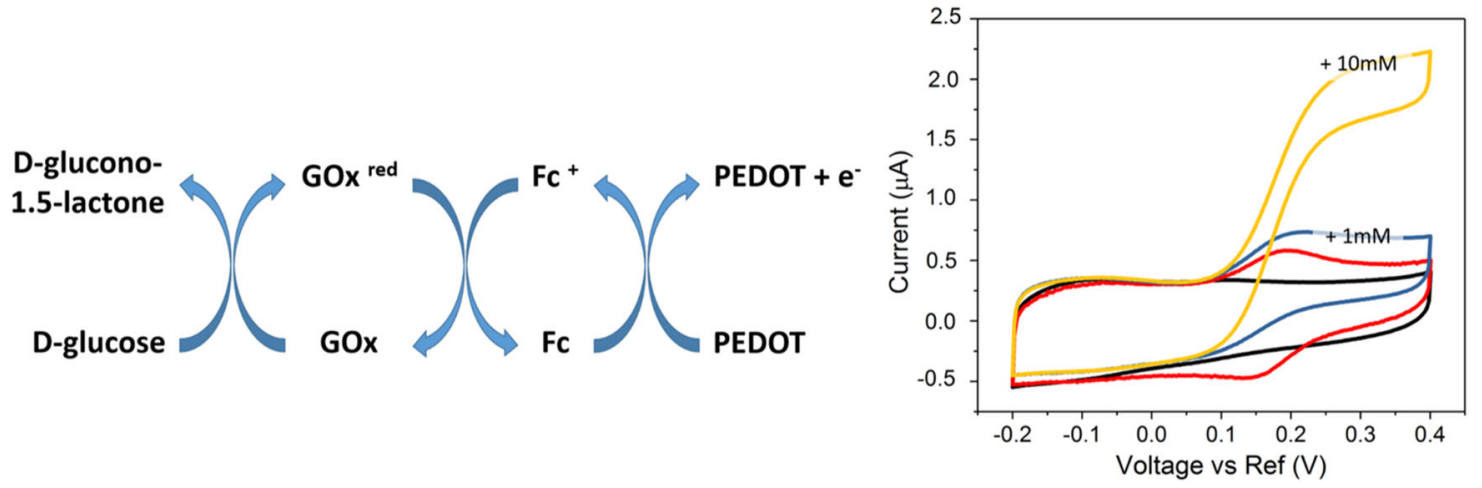

(e)

Fig. 2 SEM images of PEDOT:PSS film printed on paper $\mathbf{a}$, covered with 6 printed layers of the biological ink $\mathbf{b}$, and 2 layers of nafion $\mathbf{c}$. The scale bar is $1 \mu \mathrm{m}$. d Mechanism of the enzymatic reaction. Upon reaction with glucose, the reduced enzyme is re-oxidized via Fc ${ }^{+} / F_{c}$ couple. The electron mediator then transfers one electron to PEDOT:PSS. e Cyclic voltammetry (CV) curves of the biosensor comprising 6 printed layers of the biological layer measured in PBS (red lines) and in the presence of glucose (1 $\mathrm{mM}$ and $10 \mathrm{mM}$, blue and yellow lines, respectively). The black line is the CV curve of PEDOT:PSS before the deposition of the biological layer. The scan rate is $20 \mathrm{mV} / \mathrm{s}$. The device was not coated with nafion

Spectroscopy (XPS) C 1s spectra show characteristics peaks representative of each layer: for instance, $\mathrm{C}-\mathrm{O}$ for PEDOT:PSS, $\mathrm{C}=\mathrm{O}$ for the biological ink (chitosan) and $\mathrm{C}-\overline{\mathrm{F}}$ for nafion (Figure $\mathrm{S} 1 \mathrm{a})$. The peaks located in $\mathrm{N}$ 1s region only appear after the biological layer is printed (Figure S1b). S 2p spectrum undergoes changes upon nafion addition due to the $\mathrm{SO}_{3}$ bonds (Figure S1c), confirming the layer-by-layer integration of each component on top of PEDOT:PSS.

The mechanism of glucose detection based on the enzyme/ mediator complex involves a cycle of electrochemical reactions at the surface of the working electrode as depicted Fig. 2d. Upon reacting with glucose, GOx gets reduced. The reduced enzyme cycles back via the ferrocene/ferricenium $\left(\mathrm{Fc} / \mathrm{Fc}^{+}\right)$ion couple which mediates electron transfer from the active sites of GOx to the underlying PEDOT:PSS electrode. This reaction causes a change in the current flowing between the working and counter electrodes, proportional to the concentration of glucose. Figure 2e shows the cyclic voltammetry (CV) curves of the PEDOT:PSS electrode, before and after its modification with the Fc/GOx film as well as in the presence of glucose in the electrolyte. The electrolyte, phosphate buffered saline solution (PBS, pH 7.4), is placed on top of the active area of the sensor. The well-defined and symmetric peaks at ca. $0.2 \mathrm{~V}$ and ca. $0.15 \mathrm{~V}$ (anodic and cathodic, respectively) of the biofunctionalized PEDOT:PSS are characteristic of Fc (compare black and red lines in Fig. 2e). The biological ink here was printed as 6 successive layers and we observed a direct correlation between the number of printed layers with the amplitude of Fc peaks (Figure S2a). Upon addition of glucose into the PBS solution, we observe a drastic increase in the anodic current, evidencing effective immobilization of GOx, its reaction with glucose and communication with Fc and PEDOT:PSS (Fig. 2e, S2b). The current increases further with more glucose present in the solution (Fig. 2e). These results agree well with the previously reported work of Yun et al. who developed a printed PEDOT:PSS electrode functionalized with GOx and horseradish peroxidase. ${ }^{18}$ This device detected glucose in a measurement solution containing the mediator ferrocene methanol (FcMeOH) using external $\mathrm{Pt}$ and $\mathrm{Ag} / \mathrm{AgCl}$ electrodes as counter and reference electrodes, respectively. In our approach, all the electrodes necessary to operate the sensor are made of PEDOT:PSS printed at a single step, as well as other components printed in an automated fashion. To evaluate the performance of PEDOT:PSS as a reference electrode, we measured the open circuit potential of a PEDOT:PSS film vs. printed PEDOT:PSS reference electrode in PBS and saliva as a function of time (Figure S3). The potential of the electrode quickly stabilized in both media and remained constant over the course of the measurements. In order to verify the accuracy of our all-polymer sensor, we tested the alternative configuration which includes counter and reference electrodes printed from a commercially available $\mathrm{Ag}$ ink and post treated with a bleaching solution for chlorination. Comparing the CV curves of these devices with those of all-polymer sensors, we observe almost identical responses to glucose (Figure S4a-b).

Complex biological fluids such as saliva contain molecules other than glucose that are known to interact electrically with the conducting polymer. These endogenous interferents are the main obstacles in electrochemical detection of glucose as they lead to inaccurate read-outs. For example, uric acid and ascorbic acid have their oxidation potentials within the operation potential of our sensor $(V=0.35 \mathrm{~V})$ and can thus be oxidized by PEDOT:PSS. In order to overcome the interference, cation exchange membranes comprising materials like chitosan and nafion are typically coated on top of sensing electrodes as they prevent negatively charged species from reaching the electrode surface. ${ }^{8}$ Without such an 
(a)

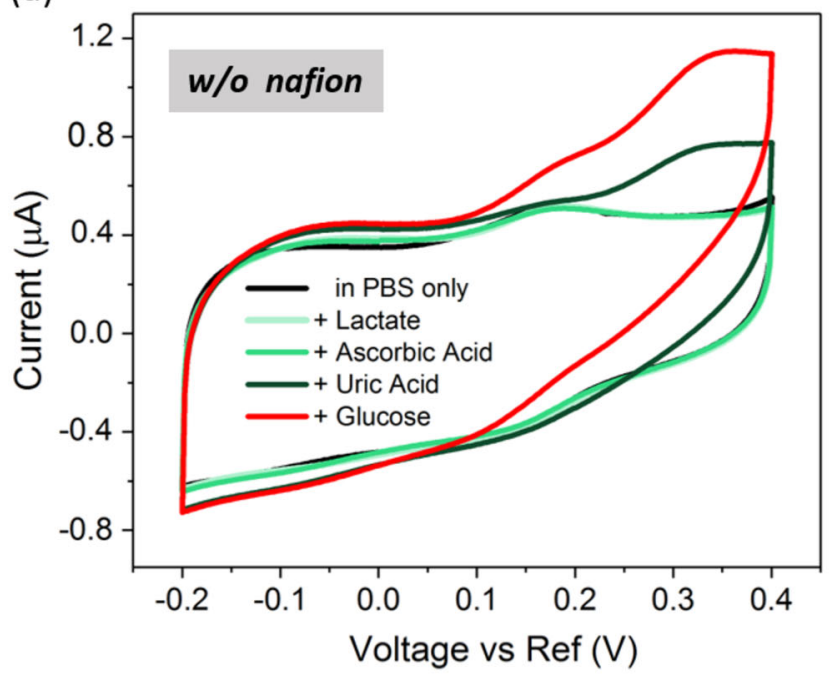

(c)

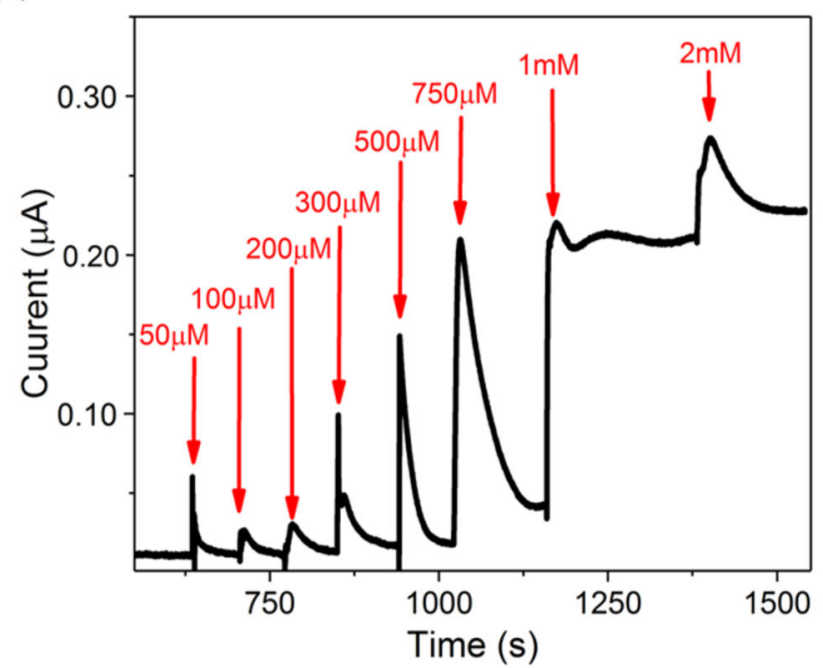

(b)

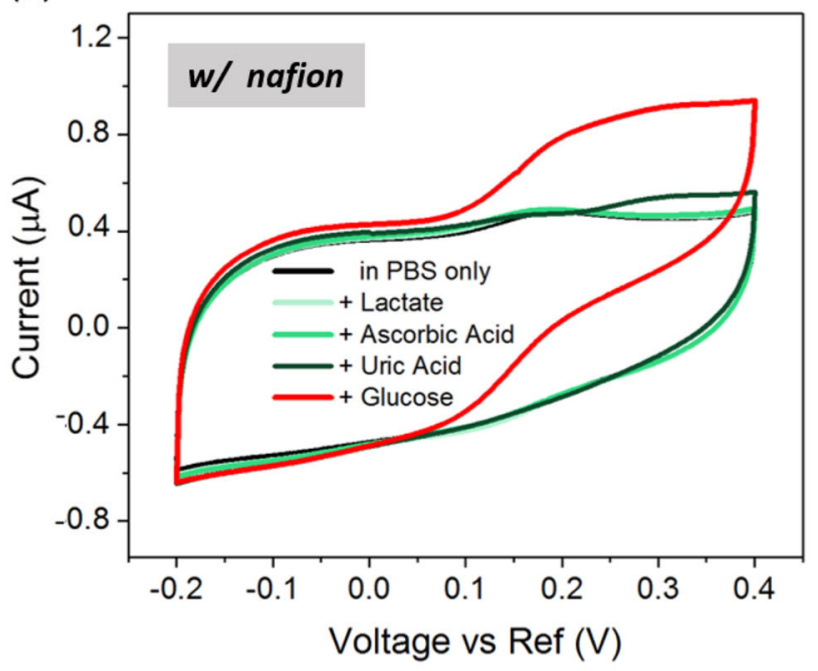

(d)

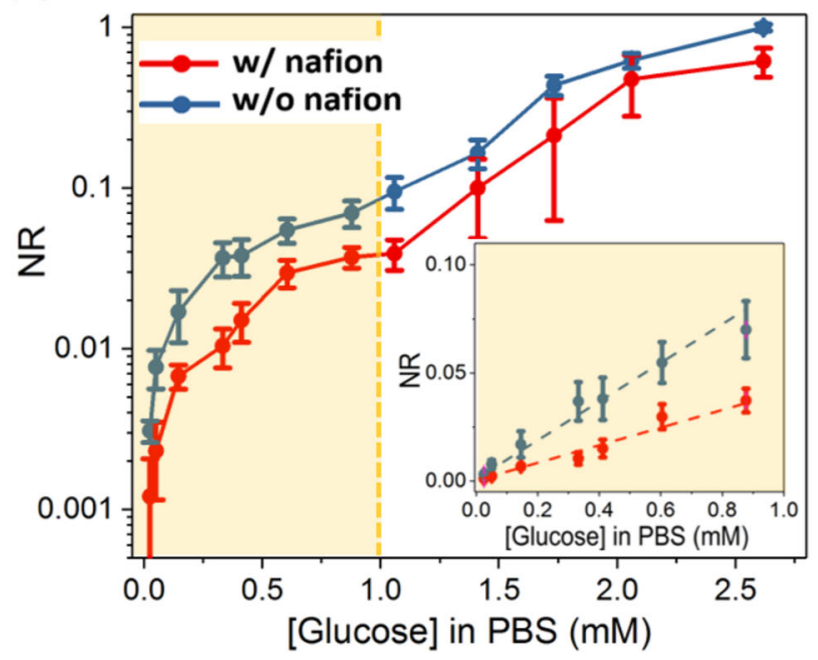

Fig. 3 CV curves of a printed sensor $\mathbf{a}$ without and $\mathbf{b}$ with nafion encapsulation (2 printed layers) in the presence of various chemical species in PBS solution. In the interference tests, the concentrations of these compounds in PBS are $2 \mathrm{mM}$ for lactate, $0.01 \mathrm{mM}$ for ascorbic acid, and $0.15 \mathrm{mM}$ for uric acid while glucose concentration is $1 \mathrm{mM}$. Note that the compounds were added successively to the solution, from lactate to glucose. c Current-time characteristics of the sensor in response to different concentrations of glucose added successively into the measurement solution (PBS). The arrows highlight the times at which glucose was introduced into PBS. The applied voltage is $0.25 \mathrm{~V}$ versus PEDOT:PSS reference electrode. $\mathbf{d}$ Normalized response (NR) of the sensor to glucose in PBS with (red curve) and without nafion (blue curve). The inset is the magnified calibration curve in linear scale. Data represent average read-outs from three different devices

encapsulation layer, our device is in fact sensitive to the most common interfering compounds, namely lactate, ascorbic acid and uric acid, all introduced to the measurement solution in the concentration range relevant to their physiological levels in saliva, that is $2 \mathrm{mM}_{1}^{5} 0.01 \mathrm{mM}^{33}$ and $0.15 \mathrm{mM}_{1}^{34}$ respectively. Among these molecules, the sensor is most sensitive to uric acid (Fig. 3a). With uric acid, the CV curve exhibits an increase in the anodic peak at the same potential range as for glucose, while the response to lactate or ascorbic acid is rather negligible. Nevertheless, the presence of a $190 \mathrm{~nm}$ thick (2 printed layers) nafion membrane on top of the biological coating eliminates the diffusion of uric acid and reduces the current response by $84 \%$ (Fig. 3a, b). In order to evaluate the sensor performance, we monitored the real-time changes in the current of the sensor upon additions of cumulative concentrations of glucose (from $50 \mu \mathrm{M}$ to $2 \mathrm{mM}$ ) into the measurement solution (Fig. 3c). To account for batch-to-batch variations in the current output of the sensors, we normalize the current response to glucose with the read-out signal at zero glucose concentration and the maximum possible output of the device. The normalized response (NR) of the sensor is calculated from:

$\mathrm{NR}=\frac{\left(I_{\mathrm{d}}-I_{0}\right)}{\left(I_{\mathrm{m}}-I_{0}\right)}$,

where $I_{0}$ is the baseline current (i.e., the current measured after stabilization of the sensor in the absence of glucose), $I_{m}$ the maximum possible current that the readout can reach (i.e., saturation), and $I_{\mathrm{d}}$ is the current measured current at a given glucose concentration. Note that $l_{\mathrm{d}}$ reaches as stable value ca. $60 \mathrm{~s}$ after the addition of glucose, which enabled the extraction of a calibration curve. Figure $3 d$ shows the NR of the sensor as a function of glucose concentration. For concentrations between $25 \mu \mathrm{M}$ and $0.9 \mathrm{mM}$, the current increases linearly, and the sensor reaches a plateau after the introduction of $2.5 \mathrm{mM}$ of glucose. The presence of the nafion membrane somehow hinders the interactions between glucose in PBS and the biological coating, 
(a)

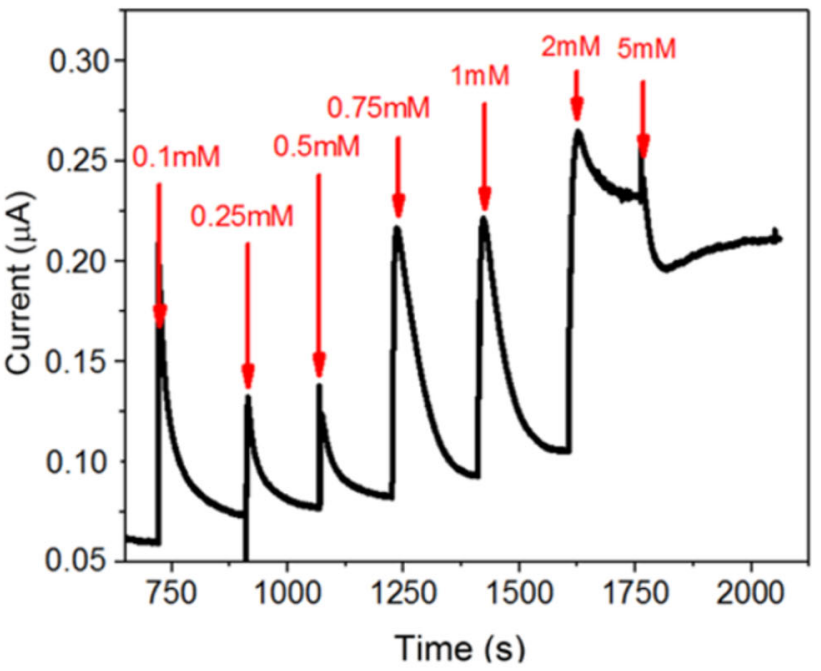

(c)

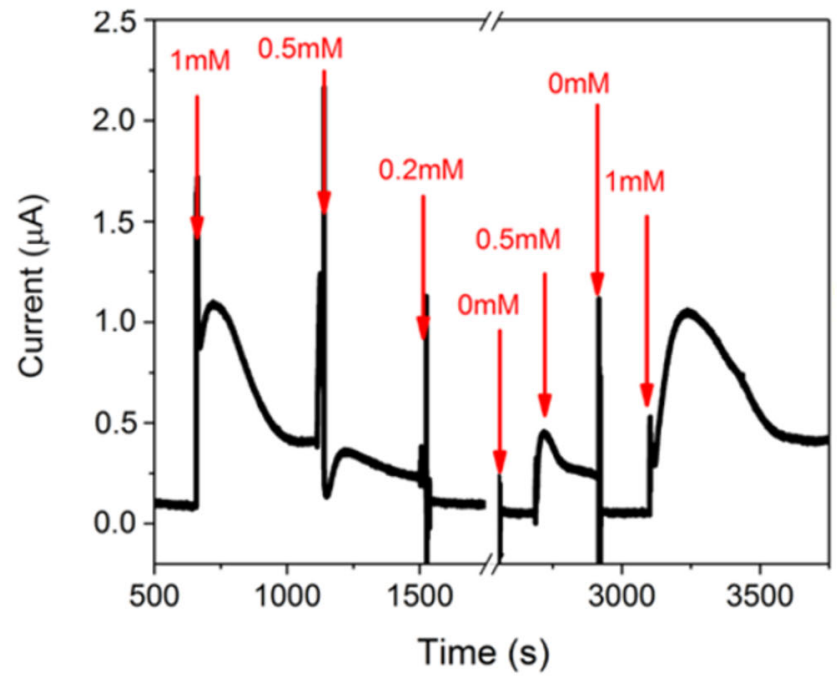

(b)

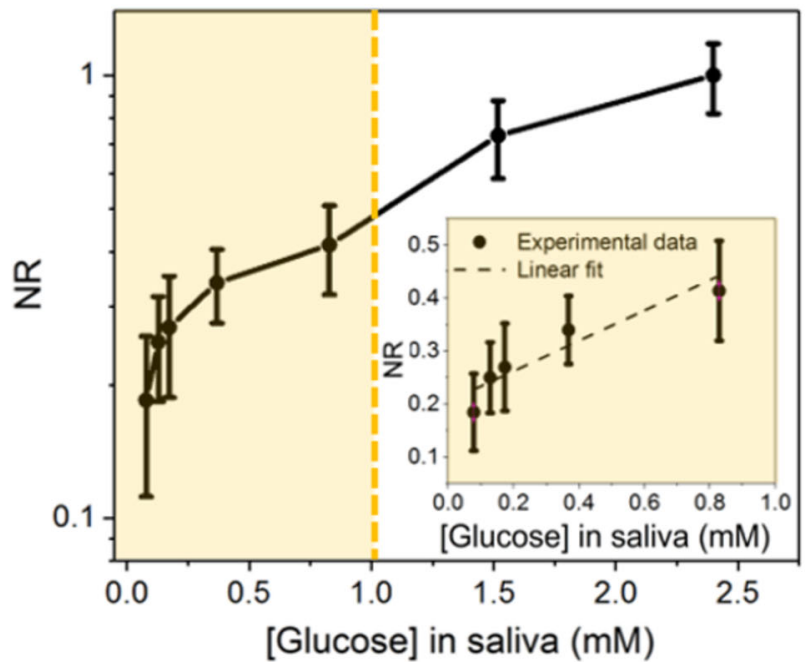

(d)

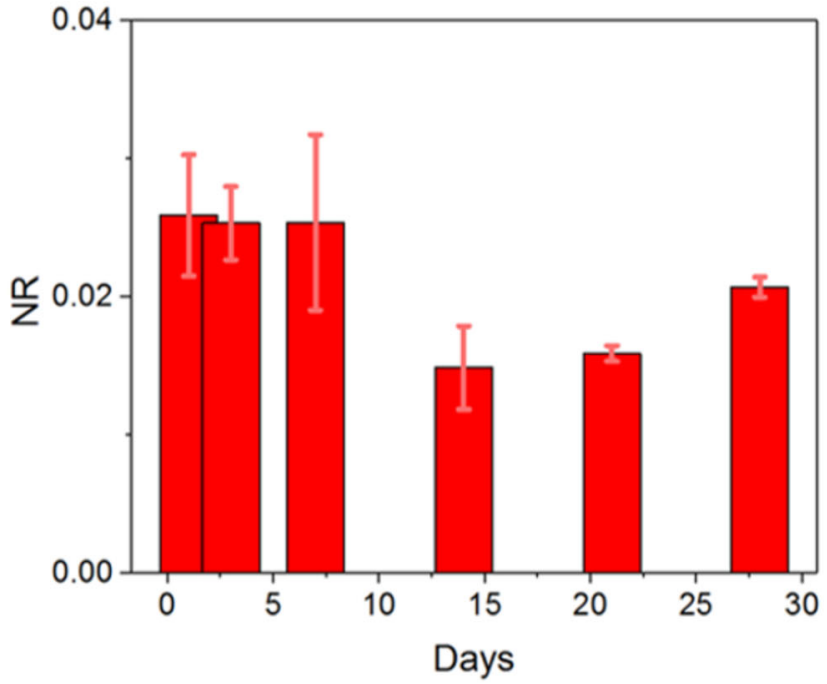

Fig. 4 a Current-time characteristics of the sensor in response to different concentrations of glucose successively added into saliva. The arrows highlight the times at which glucose was introduced into the measurement solution. The applied voltage is $0.25 \mathrm{~V}$ versus PEDOT:PSS reference electrode. $\mathbf{b}$ NR of the sensor to glucose in saliva. The highlighted area represents the range for physiologically relevant glucose concentrations. The inset is the calibration curve in linear scale magnified for low glucose concentrations with the dashed lines representing the linear fit. The error bars are extracted from the read-outs of 3 different devices. c Real-time current changes when glucose is added into or taken from the measurement solution. To decrease the concentration of glucose, a pre-determined volume of the solution is replaced with the same amount of PBS. d The performance of the sensors that have been stored for up to one month after fabrication. The glucose concentration in the measurement solution is $1 \mathrm{mM}$

resulting in a reduced $\mathrm{NR}$, yet the curve exhibits overall a similar saturation regime with a linear response to concentrations lower than $0.9 \mathrm{mM}$ (Fig. 3d). Increasing the thickness of the encapsulation layer impedes the diffusion of molecules into the PEDOT:PSS layer underneath, while we do not observe a difference between 2 or 4 layers of nafion (Figure S5a). Although the efficacy of nafion in eliminating interference comes at the expense of sensitivity and speed, both the steady-state and the time resolved characteristics of the sensors are within sufficient boundaries. In fact, the linear range of this sensor corresponds to the physiological levels of glucose in saliva, typically ranging between $20 \mu \mathrm{M}$ and $1 \mathrm{mM}$ (Fig. $3 \mathrm{~d}$, inset). ${ }^{35-38}$

To evaluate the ability of the sensor to detect glucose in saliva, we collected a saliva sample from a healthy, non-diabetic volunteer who was asked to fast $12 \mathrm{~h}$ before the extraction of saliva. Using a commercially available glucose assay kit, we found glucose in this sample at a concentration of $28 \mu \mathrm{M}$. Figure S5b shows that the CV curve of the sensor differs when measured in saliva compared to PBS due to the presence of glucose and other interferents. Since the glucose concentration in this sample is low, we decided to use it as the buffer solution to calibrate the sensor. We enriched the saliva with additions of glucose to mimic the glucose concentration range typical for diabetic patients. ${ }^{35}$ We recorded the chronoamperometric signal of the device in response to cumulative additions of glucose as depicted in Fig. 4a. The device has a linear response to glucose within the range relevant to the glucose concentrations of non-diabetic and diabetic saliva (from $28 \mu \mathrm{M}$ to $0.85 \mathrm{mM}$ ) (Fig. 4b). ${ }^{35-38}$ Diabetic patients are advised to keep their blood glucose levels close to the target range below $7 \mathrm{mM}$ (fasting). ${ }^{39,40}$ Recent studies have demonstrated a significant positive correlation between the concentration of glucose in saliva and blood for healthy and 
diabetic patients and substantiated the role of saliva as a noninvasive diagnostic tool. ${ }^{35-38}$ In saliva, the equivalent of this critical concentration is ca. $0.13 \mathrm{mM}^{35}$ As such, the sensor is relevant for diabetes treatment and reducing the risk of a coma. The sensor response is modulated only by the dose, it is reversible and independent of how we introduce glucose into the solution: the device shows the same read-out to a particular glucose concentration regardless of whether it is exposed to first low or high concentrations of glucose (Fig. 4c). For daily use, the paperbased electronics can be easily integrated with a portable miniaturized measurement system wherein the sensor is placed and electrically contacted without any wires (Figure S6a). The system then transfers the read-outs wirelessly to a smartphone or a tablet which correlates the current value to glucose concentration.

In order to test the reusability of these sensors, we recorded multiple CV curves in the presence of glucose including several washing steps with PBS. For these measurements, one cycle includes exposing the sensor to PBS, addition of glucose (1 mM) and then replacing the glucose solution with fresh PBS. Until the fourth cycle, we measure a stable and accurate response to glucose (with 6\% error), while upon the fourth cycle, the amplitude of the oxidation peak is reduced by $45 \%$ (Figure S6b). Between the fourth and tenth cycles, we observe small changes in the peak amplitude. The reason for the sudden drop in performance can be related to the instability of the paper against vigorous washing steps as well as leakage of unbound enzyme or the mediator throughout nafion. Future studies aim to address the exact source of this loss as well as forming a more stable biological ink. Next, to evaluate the shelf life of the device, we tested the performance of devices which have been stored for $24 \mathrm{~h}$ up to 1 month after their fabrication. Once printed, these devices have been stored in sealed vacuum bags at room temperature. We recorded the response of the sensors, i.e., peak current measured at $0.25 \mathrm{~V}$, to $1 \mathrm{mM}$ of glucose (Fig. 4d). A slight reduction of the performance occurs 14 days after the device is fabricated. After one month, $80 \pm 3 \%$ of the NR recorded at the first day is maintained. Note that when the sensors were kept in the fridge at $4{ }^{\circ} \mathrm{C}$, no significant enhancement of shelf life was attained. These results suggest that rather than temperature, an air-free environment is crucial for the stability of the biological coating. We anticipate that the shelf life of the devices is limited by the immobilization conditions of the enzyme and the mediator which govern their stability. The composition of the biological layer will be further optimized (for instance by covalently attaching Fc to a polymeric network) for robustness, reproducibility and longer shelf life.

\section{CONCLUSIONS}

We showed that via inkjet-printing that enables depositing organic molecules on low-cost, eco-friendly, and recyclable paper substrates, an easy-to-use sensor can be fabricated for daily monitoring of glucose in complex biological media such as saliva. All the components of this sensor were printed as a layer-by-layer assembly, including the conducting polymer PEDOT:PSS as the electronic component, a biological film comprising of the enzyme/ mediator as well as a dielectric and encapsulation layer. The allpolymer sensor exhibits good sensitivity to glucose in saliva within a concentration range relevant to diabetes and exhibits a shelf life of one month. Although we benchmarked our platform using glucose as the target metabolite, the analyte repertoire can be expanded by simply changing the enzyme. This technology platform can encompass a variety of biorecognition elements targeting multifunctionality and other applications as well. Most importantly, the design can be adapted to enable continuous monitoring of glucose from saliva once integrated with wearable biocompatible substrates. Given its versatility and adaptability, this device concept is anticipated to provide a leap toward the next generation wearable, disposable, user-friendly, automated and affordable diagnostic tools.

\section{MATERIALS AND METHODS}

Ink formulation

To formulate the PEDOT:PSS ink, we prepared a solution including PEDOT:

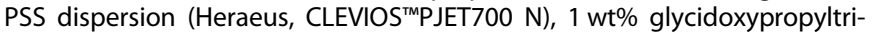
methoxysilane (GOPS, Sigma Aldrich) and $0.4 \% \mathrm{v} / \mathrm{v}$ of dodecyl benzene sulfonic acid (DBSA). GOPS was added to prevent any delamination of the conducting pattern from the paper and DBSA to improve the wettability of the ink and film formation during printing. For the preparation of the biological ink, $10 \mathrm{mg}$ of GOx (from Aspergillus Niger $>=100 \mathrm{U} \mathrm{mg}^{-1}$, Sigma Aldrich) was dissolved in $0.1 \mathrm{M}$ standard PBS solution (Sigma Aldrich). The enzyme solution was then mixed with $\mathrm{N}$-(3-dimethylaminopropyl)- $\mathrm{N}^{\prime}$ ethylcarbodiimidehydrochloride/sulfo-N-hydroxysuccinimide (EDC:NHS) $(1: 1,200 \mathrm{mM})$ solution in 2-(N-morpholino)ethanesulfonic acid (MES) buffering agent in a 5:1:1 ratio. EDC:NHS solution was prepared by first addition of EDC and $30 \mathrm{~min}$ after this, including NHS in the reaction mixture. Meanwhile, $28 \mathrm{mg}$ Chitosan (from Shrimps, Sigma Aldrich) was dissolved in $0.2 \mathrm{M}$ acetic acid, and $2.3 \mathrm{mg} / \mathrm{mL}$ of Fc (Sigma Aldrich) solution was prepared in ethanol and left for $30 \mathrm{~min}$ in an ultrasonic bath. These two solutions were then mixed thoroughly for 30 minutes. Once the Chitosan/Fc solution was ready, it was mixed with the enzyme activated solution and left overnight in the fridge at $4{ }^{\circ} \mathrm{C}$. EDC-NHS reaction conjugated the carboxyl groups of GOx to the amine groups of chitosan. As for the preparation of the anti-interference layer, nafion (Nafion 117 solution, Sigma Aldrich) was mixed with deionized water at a ratio 4:1 and its final concentration was $1 \mathrm{wt} \%$. We used a commercial dielectric ink (EMD6200, SunChemical) for the fabrication of the insulation layer.

\section{Inkjet-printing}

We used a Dimatix DMP-2800 inkjet-printer to fabricate the device. We printed 2 layers of PEDOT:PSS ink on a commercial glossy paper (ArjoWiggins). The drop spacing was $20 \mu \mathrm{m}$. Following printing, the samples were cured in a conventional oven at $160^{\circ} \mathrm{C}$ for $30 \mathrm{~min}$. The second printing step was for casting of the dielectric layer to insulate the PEDOT:PSS areas outside of the sensing and the connection areas. Upon printing 1 layer of the dielectric ink, we cured the pattern for $5 \mathrm{~min}$ in a UV/ Ozone chamber (Ossila, UV ozone cleaner). We then printed the biological ink $(2,4$, and 6 layers) and let it dry at room temperature for $24 \mathrm{~h}$. Finally, nafion was printed on top of the sensing area. To verify the accuracy of PEDOT:PSS electrodes for biosensing, we also printed 2 layers of silver as reference and counter electrodes (ink DGP HR, ANP. Co). The silver coatings were treated by a bleach solution (Clorox, 10\%) drop casted on the patterns to form $\mathrm{Ag} / \mathrm{AgCl}$.

\section{Scanning electron microscopy (SEM)}

SEM images of printed sensor layers were acquired using FEI Nova nano microscope at accelerating voltages of $3 \mathrm{kV}$ and working distances of $4.5 \mathrm{~mm}$. The samples were coated with $3 \mathrm{~nm}$ thick iridium and mounted on aluminum stubs using aluminum tape for imaging. FIB-SEM for crosssection image was prepared on an FEI Helios NanoLab 400 S FIB/SEM dualbeam system equipped with $\mathrm{a} \mathrm{Ga}^{+}$ion source. C/Pt layers were deposited on the surface region of interest by Electron \& lon beam for sample protection.

\section{X-ray photoelectron spectroscopy (XPS)}

XPS experiments were performed on a KRATOS Analytical AMICUS instrument equipped with an achromatic Al Ka X-ray source $(1468.6 \mathrm{eV})$. Typically, the source was operated at voltage of $10 \mathrm{kV}$ and current of $10 \mathrm{~mA}$ generating 100 Watts. The high-resolution spectra were acquired using a step of $0.1 \mathrm{eV}$. The pressure in the analysis chamber was in the range of $\times 10^{-7} \mathrm{~Pa}$ during the whole measurement time.

\section{Device characterization}

Cyclic voltammetry (CV) and chronoamperometry measurements were performed using a potentiostat-galvanostat (Metrohm Autolab B.V.) and the data were collected with the NOVA software. CV scans were recorded in PBS solution from -0.2 to $0.4 \mathrm{~V}$ vs. PEDOT:PSS reference electrode, unless otherwise stated. For chronoamperometry measurements, the 
voltage was set at $0.25 \mathrm{~V}$ vs. reference electrode. All the data shown in this work were collected from devices that were used $24 \mathrm{~h}$ after being printed unless stated otherwise.

\section{Chronoamperometric measurements for the calibration of the sensor}

To acquire the calibration curves, the applied potential was set to $0.25 \mathrm{~V}$ vs. reference electrode and interval time for data collection was $0.1 \mathrm{~s}$. A PDMS well was placed on top of the active area $\left(4 \times 4 \mathrm{~mm}^{2}\right)$ to confine the electrolyte (Figure S7). The total volume of the PBS solution in this well was $30 \mu \mathrm{L}$. Different concentrations of glucose (Sigma Aldrich) in PBS were added to this solution at a 1:10 ratio of the total volume. The glucose concentration varied between $25 \mu \mathrm{M}$ and $2.6 \mathrm{mM}$. We measured the response of three different devices (current-time curves) to each added concentration of glucose. During the calibration measurements, it was ensured that the baseline current (in PBS, no glucose) was stabilized before the addition of glucose. For the experiments using saliva, we collected the saliva of a healthy volunteer after fasting $(12 \mathrm{~h})$ and filtered the solution with a filter of $1 \mu \mathrm{m}$ pore size. We measured the glucose concentration in this sample using a commercial Glucose Assay Kit (GAGO-20, Sigma Aldrich) using a spectrophotometer (Promega). To mimic variations of glucose in physiological saliva, different concentrations of glucose were added to this sample and measured by the printed sensor. All protocols and procedures involving human saliva were approved by the KAUST Institutional Biosafety and Bioethics Committee (IBEC). The volunteers provided signed consent to participate in the study.

\section{DATA AVAILABILITY}

All experimental data generated or analyzed during this work are included in the article and the Supplementary Information Files.

\section{ACKNOWLEDGEMENTS}

E.B. and S.I. thank Prof. Valerio Orlando and his team for helping with the spectrophotometer measurements and Nini Wei at Imaging and Characterization Core labs at KAUST for FIB analysis.

\section{AUTHOR CONTRIBUTIONS}

S.I. conceived and coordinated the research. E.B. designed and performed the experiments with contributions from D.B. E.B. fabricated the device. E.B., S.W., and A. M.P. analyzed the data. S.W. performed SEM and XPS measurements. K.N.S. helped with the acquisition system. E.B. and S.I. wrote the manuscript. All authors revised the manuscript.

\section{ADDITIONAL INFORMATION}

Supplementary information accompanies the paper on the npj Flexible Electronics website (https://doi.org/10.1038/s41528-018-0044-y).

Competing interests: A patent application about the metabolite sensor fabrication and function was filed by KAUST with E.B. and S.I as the inventors, pending with the application number of $62 / 674,374$. The remaining authors declare no competing interests.

Publisher's note: Springer Nature remains neutral with regard to jurisdictional claims in published maps and institutional affiliations.

\section{REFERENCES}

1. Sharma, S., Zapatero-Rodríguez, J., Estrela, P. \& O'Kennedy, R. Point-of-care diagnostics in low resource settings: present status and future role of microfluidics. Biosensors 5, 577-601 (2015).

2. Estimated number diabetics worldwide 2017 | Statistic. Statista https://www. statista.com/statistics/271442/number-of-diabetics-worldwide/. Accessed 19th March 2018.

3. Wang, H.-C. \& Lee, A.-R. Recent developments in blood glucose sensors. J. Food Drug Anal. 23, 191-200 (2015).

4. Soni, A. \& Jha, S. K. A paper strip based non-invasive glucose biosensor for salivary analysis. Biosens. Bioelectron. 67, 763-768 (2015).
5. Pappa, A.-M. et al. Organic transistor arrays integrated with finger-powered microfluidics for multianalyte saliva testing. Adv. Healthc. Mater. 5, 2295-2302 (2016).

6. Lee, H., Hong, Y. J., Baik, S., Hyeon, T. \& Kim, D. Enzyme-based glucose sensor: from invasive to wearable device. Adv. Healthc. Mater. 7, 1701150-1701164 (2018).

7. Gao, W. et al. Fully integrated wearable sensor arrays for multiplexed in situ perspiration analysis. Nature 529, 509-514 (2016).

8. Lee, $\mathrm{H}$. et al. Wearable/disposable sweat-based glucose monitoring device with multistage transdermal drug delivery module. Sci. Adv. 3, e1601314 (2017).

9. Wang, J. Electrochemical glucose biosensors. Chem. Rev. 108, 814-825 (2008).

10. Pappa, A.-M. et al. Organic electronics for point-of-care metabolite monitoring. Trends Biotechnol. 36, 45-49 (2017).

11. Kim, J., Campbell, A. S. \& Wang, J. Wearable non-invasive epidermal glucose sensors: a review. Talanta 177, 163-170 (2018).

12. Wang, J. Carbon-nanotube based electrochemical biosensors: a review. Electroanalysis 17, 7-14 (2005).

13. Grieshaber, D., MacKenzie, R., Voeroes, J. \& Reimhult, E. Electrochemical biosensors-sensor principles and architectures. Sensors 8, 1400-1458 (2008).

14. Calvert, P. Inkjet printing for materials and devices. Chem. Mater. 13, 3299-3305 (2001).

15. Setti, L. et al. An amperometric glucose biosensor prototype fabricated by thermal inkjet printing. Biosens. Bioelectron. 20, 2019-2026 (2005).

16. Abe, K., Suzuki, K. \& Citterio, D. Inkjet-printed microfluidic multianalyte chemical sensing paper. Anal. Chem. 80, 6928-6934 (2008).

17. Delaney, J. T., Smith, P. J. \& Schubert, U. S. Inkjet printing of proteins. Soft Matter 5, 4866-4877 (2009).

18. Yun, Y. H. et al. A glucose sensor fabricated by piezoelectric inkjet printing of conducting polymers and bienzymes. Anal. Sci. Int. J. Jpn Soc. Anal. Chem. 27, 375 (2011).

19. Beni, V. et al. Printed electrochemical instruments for biosensors. ECS J. Solid State Sci. Technol. 4, S3001-S3005 (2015).

20. Scheiblin, G. et al. Screen-printed organic electrochemical transistors for metabolite sensing. MRS Commun. 5, 507-511 (2015)

21. Imani, S. et al. A wearable chemical-electrophysiological hybrid biosensing system for real-time health and fitness monitoring. Nat. Commun. 7, 11650 (2016).

22. Bihar, E. et al. A disposable paper breathalyzer with an alcohol sensing organic electrochemical transistor. Sci. Rep. 6, 27582 (2016).

23. Li, L. et al. All Inkjet-printed amperometric multiplexed biosensors based on nanostructured conductive hydrogel electrodes. Nano Lett. (2018). https://doi. org/10.1021/acs.nanolett.8b00003

24. Inal, S., Malliaras, G. G. \& Rivnay, J. Benchmarking organic mixed conductors for transistors. Nat. Commun. 8, 1767 (2017).

25. Zucca Alessandra et al. Tattoo conductive polymer nanosheets for skin-contact applications. Adv. Healthc. Mater. 4, 983-990 (2015).

26. Bihar, E. et al. Fully printed electrodes on stretchable textiles for long-term electrophysiology. Adv. Mater. Technol. 2, 1600251-1600256 (2017).

27. Ferrari, L. M. et al. Ultraconformable temporary tattoo electrodes for electrophysiology. Adv. Sci. 5, 1700771-1700782 (2018).

28. Bihar, E. et al. Fully printed all-polymer tattoo/textile electronics for electromyography. SFlex. Print. Electron. 3, 034004 (2018).

29. Nilsson, D., Kugler, T., Svensson, P.-O. \& Berggren, M. An all-organic sensor-transistor based on a novel electrochemical transducer concept printed electrochemical sensors on paper. Sens. Actuators B Chem. 86, 193-197 (2002).

30. Tobjörk, D. \& Österbacka, R. Paper electronics. Adv. Mater. 23, 1935-1961 (2011)

31. Bihar, E. et al. Inkjet-printed PEDOT: PSS electrodes on paper for electrocardiography. Adv. Healthc. Mater. 6, 1601167-1601171 (2017).

32. Yuan, C.-J., Hsu, C.-L., Wang, S.-C. \& Chang, K.-S. Eliminating the interference of ascorbic acid and uric acid to the amperometric glucose biosensor by cation exchangers membrane and size exclusion membrane. Electroanalysis 17, 2239-2245 (2005).

33. Mäkilä, E. \& Kirveskari, P. A study of ascorbic acid in human saliva. Arch. Oral Biol. 14, 1285-1292 (1969).

34. Inoue, K., Namiki, T., Iwasaki, Y., Yoshimura, Y. \& Nakazawa, H. Determination of uric acid in human saliva by high-performance liquid chromatography with amperometric electrochemical detection. J. Chromatogr. B Anal. Technol. Biomed. Life Sci. 785, 57-63 (2003).

35. Abikshyeet, P., Ramesh, V. \& Oza, N. Glucose estimation in the salivary secretion of diabetes mellitus patients. Diabetes Metab. Syndr. Obes. 5, 149-154 (2009).

36. Kumar, S., Padmashree, S. \& Jayalekshmi, R. Correlation of salivary glucose, blood glucose and oral candidal carriage in the saliva of type 2 diabetics: a case-control study. Contemp. Clin. Dent. 5, 312 (2014).

37. Gupta, S. et al. Correlation of salivary glucose level with blood glucose level in diabetes mellitus. J. Oral Maxillofac. Pathol. 21, 334-339 (2017) 
38. Naing, Cho \& Mak, JoonWahC. Salivary glucose in monitoring glycaemia in patients with type 1 diabetes mellitus: a systematic review. J. Diabetes Metab. Disord. 16, 2251-6581 (2017).

39. Wustoni, S., Savva, A., Sun R., Bihar E. \& Inal, S. Enzyme-free detection of glucose with a hybrid conductive gel electrode. Adv. Mater. Interfaces https://doi.org/ 10.1002/admi.201800928 (2018).

40. The Global Diabetes Community, https://www.diabetes.co.uk/diabetes_care/ blood-sugar-level-ranges.html. Accessed October 2018.

Open Access This article is licensed under a Creative Commons Attribution 4.0 International License, which permits use, sharing, adaptation, distribution and reproduction in any medium or format, as long as you give appropriate credit to the original author(s) and the source, provide a link to the Creative Commons license, and indicate if changes were made. The images or other third party material in this article are included in the article's Creative Commons license, unless indicated otherwise in a credit line to the material. If material is not included in the article's Creative Commons license and your intended use is not permitted by statutory regulation or exceeds the permitted use, you will need to obtain permission directly from the copyright holder. To view a copy of this license, visit http://creativecommons. org/licenses/by/4.0/.

(c) The Author(s) 2018 